Www.jmscr.igmpublication.org

Index Copernicus Value: 79.54

ISSN (e)-2347-176x ISSN (p) 2455-0450

crossref DOI: https://dx.doi.org/10.18535/jmscr/v7i6.21

Journal Of Medical Science And Clinical Research

IGM Publication

An official Publication of IGM Publication

\title{
Correlation of Supraspinatus Tears and Tendinosis with Acromion Morphology and Acromioclavicular Arthritis Based on Conventional MRI and CT
}

\author{
Authors
}

\section{Dr Amit Achyut Ban*, Dr Mahammadhusen Taiyabali Makada, Dr Nandini Umeshkumar Bahri}

Department of Radiodiagnosis, Shri M.P Shah Medical College and Guru Gobind Singh Hospital, Jamnagar, Gujarat, India - 361008

*Corresponding Author

Dr Amit Achyut Ban

Prasad Hospital, Borban Area, Behind SP office, Govardhan Ghat Road, Taluka, District, Nanded,

Maharashtra - 431601, India

\begin{abstract}
Background: In consideration of supraspinatus tears and tendinosis, acromion morphology forms a most important risk factor along with acromioclavicular joint arthritis. Utilisation of the pathological phenomenon caused by the acromial changes can lead to identification of etiology for supraspinatus tears and tendinosis.
\end{abstract}

Aim: To evaluate the association of supraspinatus tears and tendinosis with acromion morphology and acromio-clavicular arthritis using conventional MRI and CT.

Material and Methods: Evaluation of 100 patients with chronic shoulder pain for rotator cuff tears and correlating them with findings of acromion and acromio-clavicular joint. Dual source 16-slice Computed tomography scanner (Bright speed, GE health care, UK) and 1.5 Tesla Magnetic Resonance Imaging system (Magnetom Essenza, Siemens health care, Germany) were used for imaging. Acromio-clavicular joint, rotator cuff pathologies and acromion types were evaluated using CT and MRI. Measurement of acromiohumeral distance was done using CT.

Results: In our study, 92\% cases with acromio-clavicular joint changes showed tear/tendinosis of supraspinatus tendon. The type I and type II are more seen types in the population. However, type III acromion showed supraspinatus tears in 100\% cases and type II acromion were associated with rotator cuff tears in $80 \%$ of cases. Acromio-humeral distance $<7 \mathrm{~mm}$ was associated with $70 \%$ supraspinatus tears and remaining $30 \%$ had tendinosis. Acromio-humeral distance between 7-10 $\mathrm{mm}$ was associated with supraspinatus tears in $64 \%$ cases.

Conclusion: Acromioclavicular arthritis and type III acromion showed strong association with supraspinatus tears and tendinosis. Reduced acromio-humeral distance was an important association of supraspinatus tears.

Keywords: Acromion types, acromioclavicular arthritis, acromio-humeral distance, supraspinatus tears, impingement, MRI. 


\section{Introduction}

Acromial morphology is the single most important factor in rotator cuff impingement. Acromial factors like acromial shape, acromial spurs need to be evaluated in rotator cuff pathologies. Acromioclavicular joint arthritis is also responsible for the rotator cuff impingement predominantly involving supraspinatus tendon. Evaluation of acromial morphology and acromioclavicular joint is essential in cases of rotator cuff pathologies especially in supraspinatus tears and tendinosis.

\section{Aims and Objectives}

Use of CT and MRI to evaluate:

a. Acromion morphology and its correlation with supraspinatus tears and tendinosis.

b. Acromio-clavicular joint arthritis and its effect on supraspinatus tears and tendinosis.

c. Acromio-humeral distance and supraspinatus tears and tendinosis.

\section{Materials and Methods}

Sample size: A total of 100 patients with complaints of shoulder pain for atleast for 4-6 months duration associated with swelling, stiffness, dislocation, with or without a history of trauma to shoulder were evaluated.

\section{Inclusion Criteria}

- All patients registered in the institution.

- All patients with chronic shoulder pain.

○ Age greater than 15 years.
Equipment: MR imaging was done using 1.5 Tesla Magnetic Resonance Imaging system (Magnetom Essenza, Siemens health care, Germany) and CT was done using Dual source 16 slice Computed tomography scanner (Bright speed, GE health care, UK).

Method: Supraspinatus tear and tendinosis were looked for in all cases. Out of those with supraspinatus tears and tendinosis, acromion morphology and acromio-clavicular joint changes were evaluated. Acromion types were evaluated for all 100 cases.

Statistics: To describe about the data descriptive statistics, frequency analysis and percentage analysis were used for categorical variables. The data obtained was compared with relevant studies for detection of significance.

\section{Ethics}

This study was carried out after clearance and approval of institutional ethics committee. A written and informed consent was obtained from all the subjects.

\section{Results}

Acromion types: Our study shows type II acromion to be most common with $65 \%$ cases. The type I and type II are more seen types in the population which was consistent with study by Mohamed et al ${ }^{[3]}$ who found the type II to be most common type ( $44 \%$ cases).

Table 1: Types of Acromion [Total 100 Patients]

\begin{tabular}{|ccc|}
\hline TYPE & FREQUENCY & PERCENTAGE \\
\hline TYPE 1 & 31 & $31 \%$ \\
\hline TYPE 2 & 65 & $65 \%$ \\
\hline TYPE 3 & 4 & $4 \%$ \\
\hline TOTAL & 100 & $100 \%$ \\
\hline
\end{tabular}




\section{JMSCR Vol||07||Issue||06||Page 106-115||June}

Figure 1: Types of Acromion [Total 100 Patients]

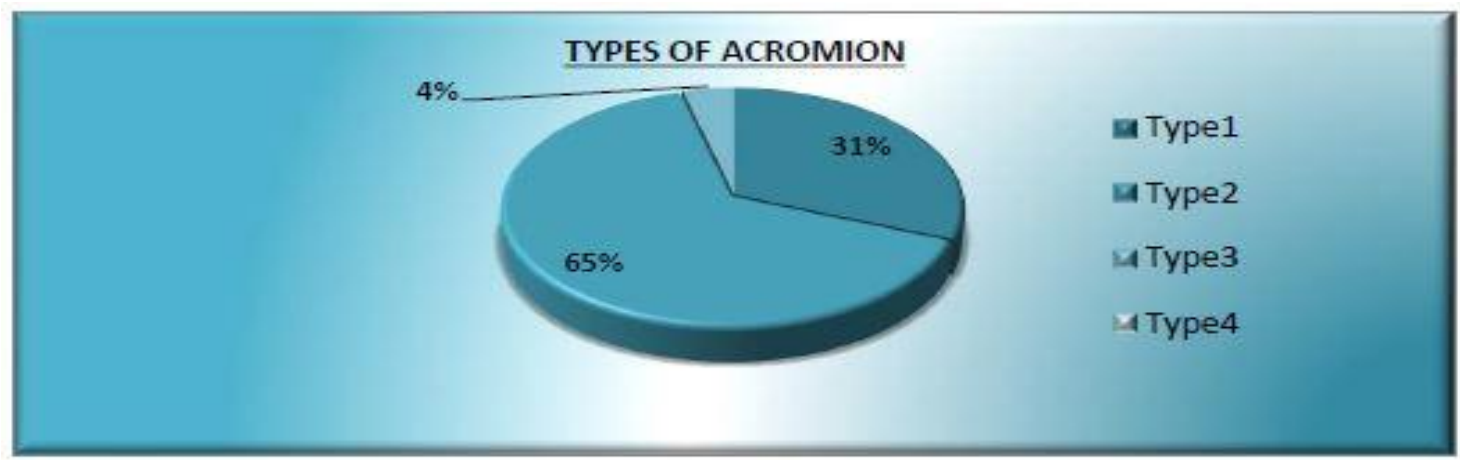

In our study, $100 \%$ cases of type III acromion were associated with rotator cuff tears whereas $80 \%$ cases with type II acromion were associated with rotator cuff tears which was consistent with Mohamed et al ${ }^{[1]}$ who found that $100 \%$ type-III acromion was associated with rotator cuff tears.

Table 2: Types of Acromion and Association with Supraspinatus Pathologies [Total 100 Patients]

\begin{tabular}{|ccccc}
\multirow{2}{*}{ ACROMION TYPE } & \multicolumn{4}{c}{ SUPRASPINATUS TENDON } \\
\cline { 2 - 4 } & NORMAL & $\%$ & TEAR/TENDINOSIS & $\%$ \\
\hline TYPE 1 & 17 & $17 \%$ & 14 & $14 \%$ \\
\hline TYPE 2 & 13 & $13 \%$ & 52 & $52 \%$ \\
\hline TYPE 3 & 0 & $0 \%$ & 4 & $4 \%$ \\
\hline TOTAL & 30 & $30 \%$ & 70 & $70 \%$ \\
\hline
\end{tabular}

Figure 2: Types of Acromion and Association with Supraspinatus Pathologies [Total 100 Patients]

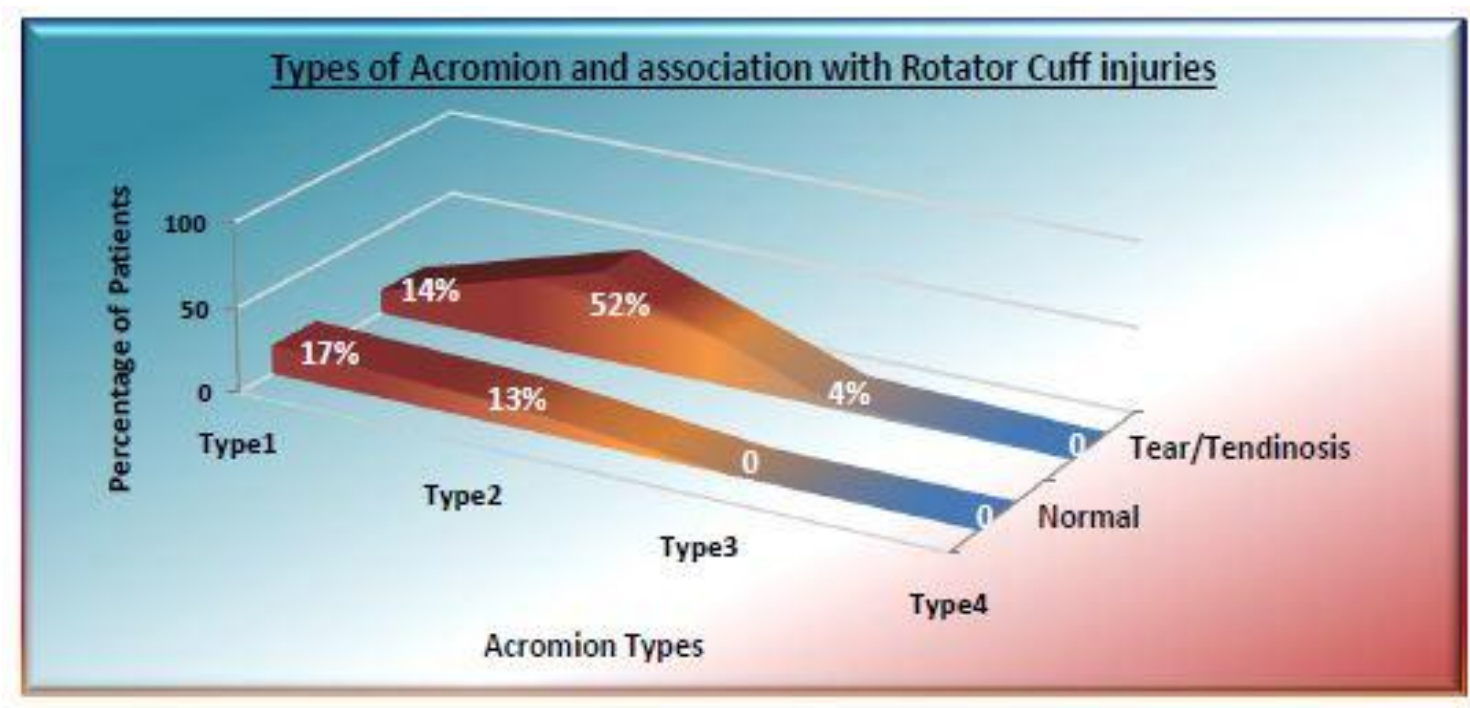

\section{Rotator cuff injuries and acromio-humeral} distance: In our study, acromio-humeral distance $<7 \mathrm{~mm}$ was associated with $70 \%$ supraspinatus tears and remaining $30 \%$ had tendinosis. Similar association was found with acromio-humeral distance between 7-10 $\mathrm{mm}$ where $64 \%$ cases were found with supraspinatus tears which was consistent with study by Saupe et $\mathrm{al}^{[2]}$ where acromio-humeral distance of $<7 \mathrm{~mm}$ was associated with supraspinatus tears in $90 \%$ cases and acromio-humeral distance between 7-10 mm with $57 \%$ had supraspinatus tears. 


\section{JMSCR Vol||07||Issue||06||Page 106-115||June}

Table 3: Supraspinatus Tendon Injuries and Acromio-Humeral Distance [N=100]

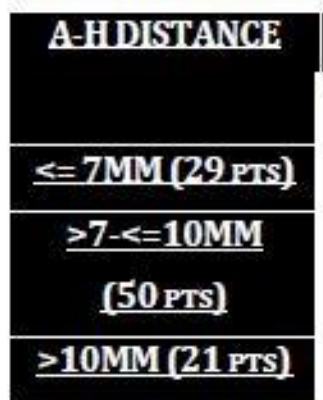

\begin{tabular}{|c|c|c|c|c|c|}
\hline \multicolumn{7}{|c|}{ SUPRASPINATUS TENDON } \\
\hline NORMAL & $\%$ & TENDINOSIS & $\%$ & TEARS & $\%$ \\
\hline 0 & $0 \%$ & 9 & $30 \%$ & 20 & $70 \%$ \\
\hline 14 & $26 \%$ & 5 & $10 \%$ & 32 & $64 \%$ \\
\hline 17 & $81 \%$ & 2 & $9.5 \%$ & 2 & $9.5 \%$ \\
\hline
\end{tabular}

Figure 3: Types of Acromion and Association with Supraspinatus Pathologies [Total 100 Patients]

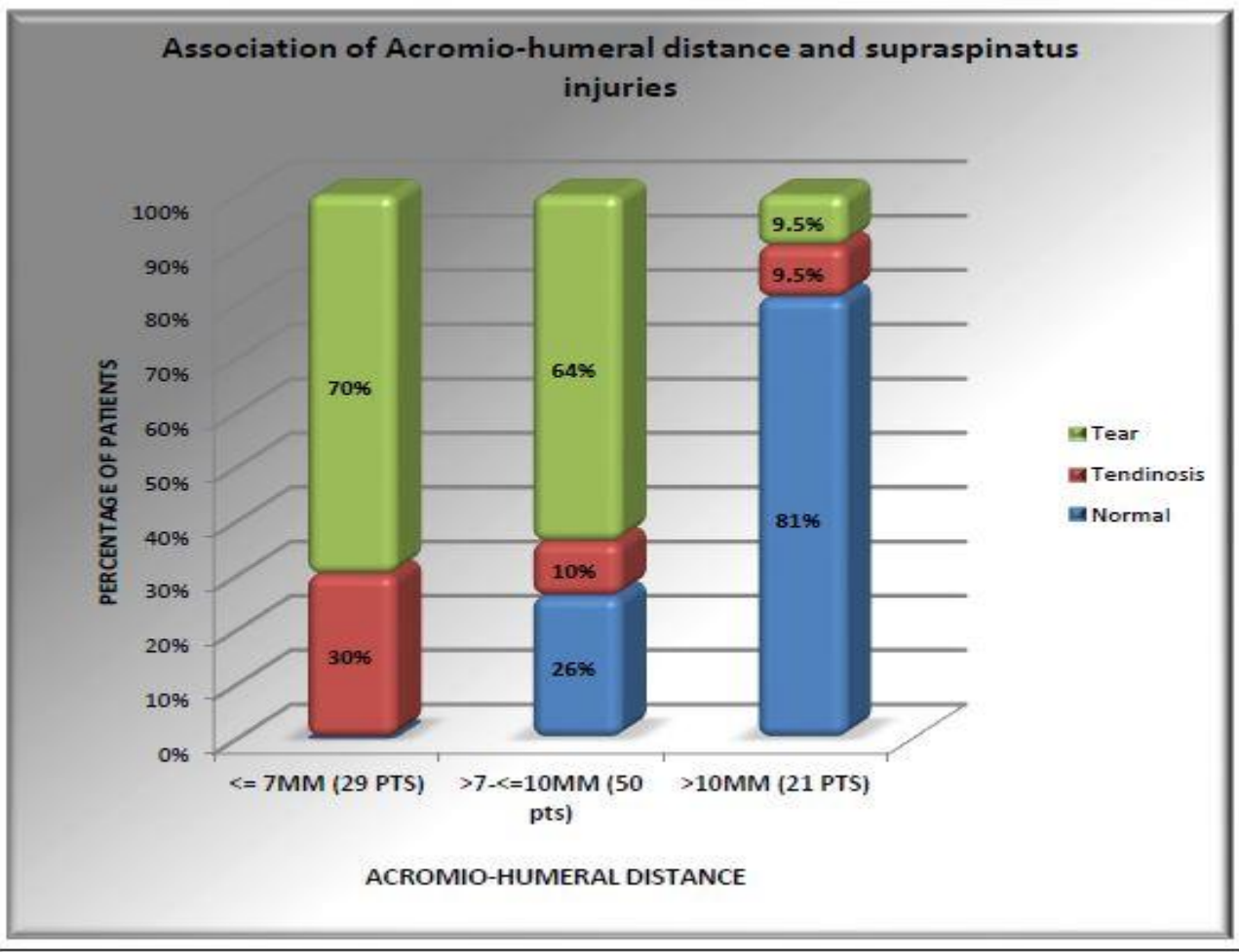

Rotator cuff injuries and acromio-clavicular joint: In our study, 92\% cases with acromioclavicular joint changes showed tear/tendinosis of supraspinatus abnormalities which was consistent with study by Needell et al ${ }^{[3]}$ where acromioclavicular joint changes were seen in $11 \%$ of the normal tendons, $39 \%$ supraspinatus tendinopathy, $68 \%$ partial teams and $86 \%$ complete teams.

Table 4: Supraspinatus Injuries and Acromio-Clavicular Joint Status [Total 100 Patients]

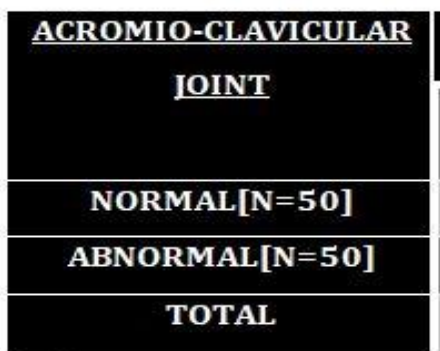

\begin{tabular}{|c|c|c|c|}
\hline \multicolumn{4}{|c|}{ ROTATOR CUFF (SUPRASPINATUS) } \\
\hline NORMAL & $\%$ & (TENDINOSIS+TEARS) & $\%$ \\
\hline 26 & $26 \%$ & 24 & $24 \%$ \\
\hline 4 & $4 \%$ & 46 & $46 \%$ \\
\hline 30 & $30 \%$ & 70 & $70 \%$ \\
\hline
\end{tabular}

N= SAMPLE SIZE 


\section{JMSCR Vol||07||Issue||06||Page 106-115||June}

Figure 4: Supraspinatus Injuries and Acromio-Clavicular Joint Status [Total 100 Patients]

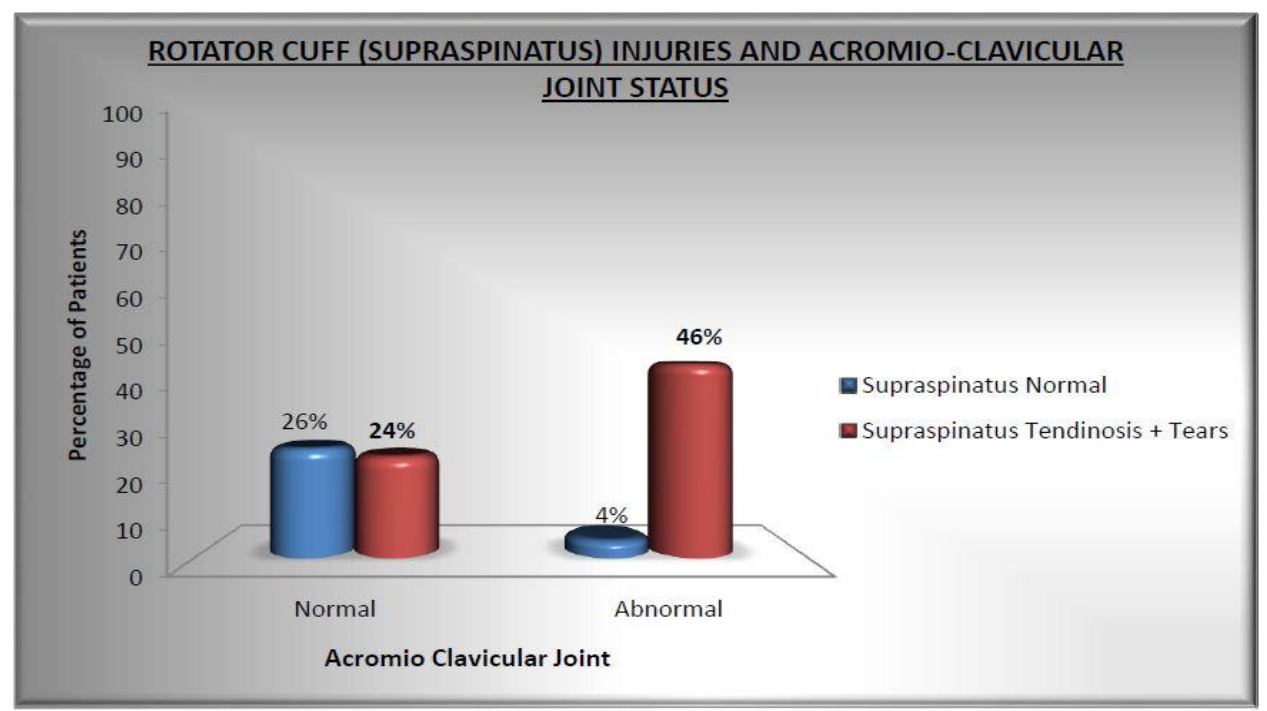

Figure 5

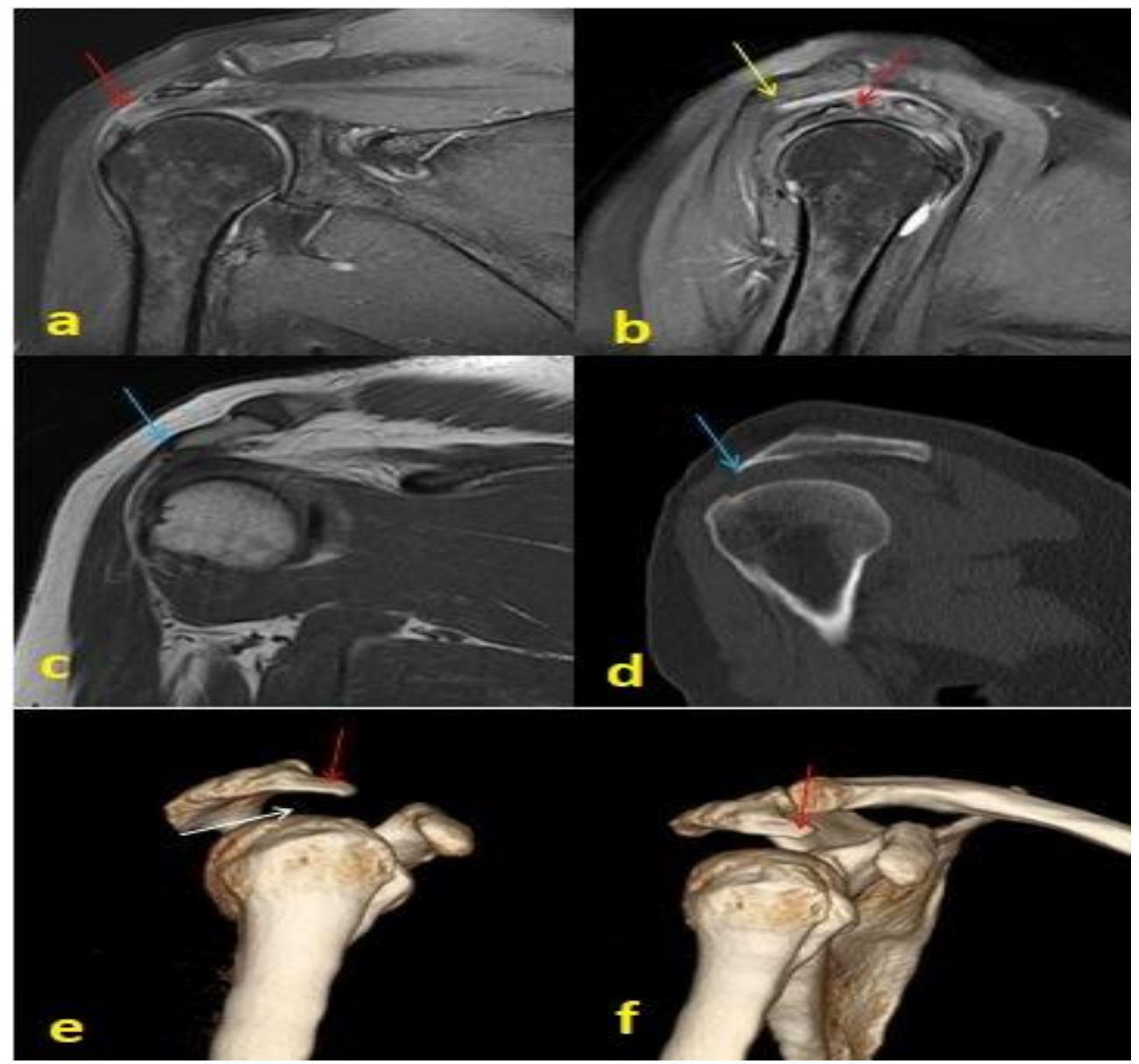

50 year-old female patient with right shoulder pain since 6 months;

[a, b] Proton-density fat-suppressed MR images of shoulder show increased signal intensity (red arrows) involving supraspinatus tendon associated with sub-acromial fluid (yellow arrow); [c, d] T1weighted MR and CT images of shoulder joint show beaking of acromion with reduced acromiohumeral distance (blue arrows); [e ,f] 3D-reformatted CT images of shoulder show type 3 acromion with beaking (red arrows) and reduced acromio-humeral distance (white arrow). 


\section{JMSCR Vol||07||Issue||06||Page 106-115||June}

Figure 6

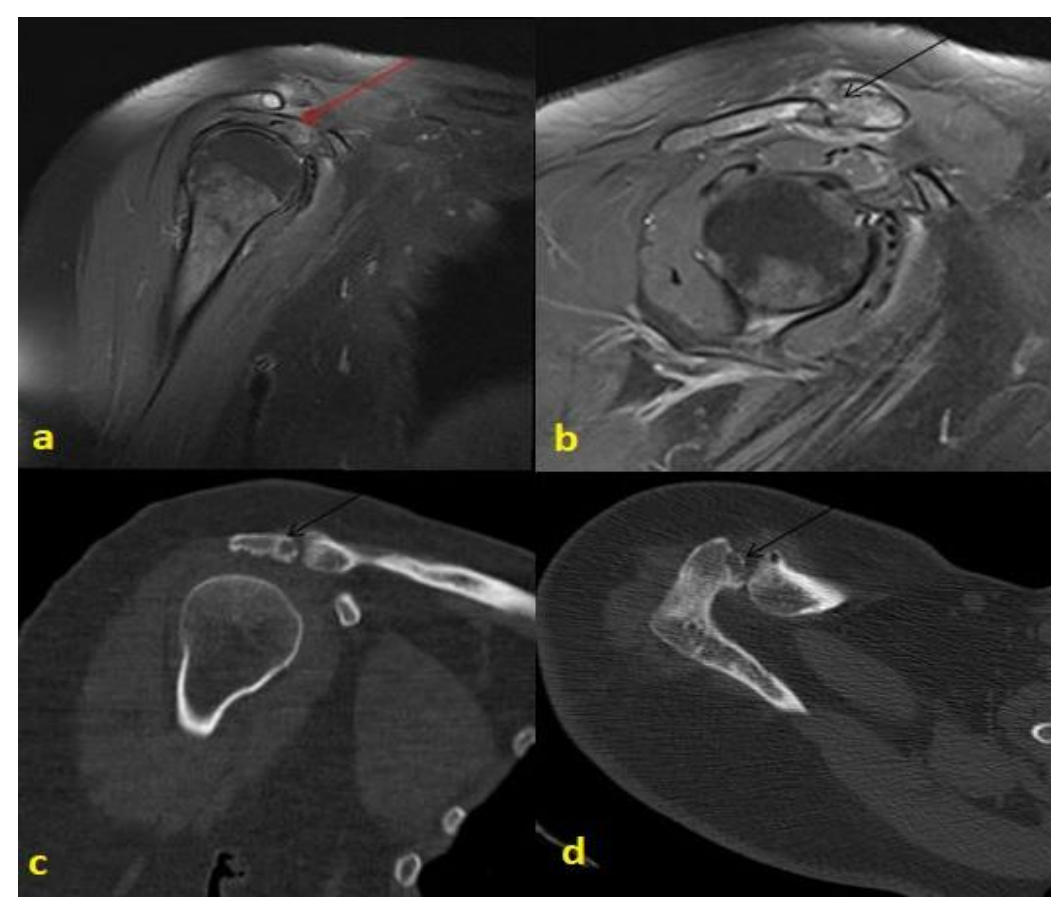

44 year-old female patient with right shoulder pain since 5 months;

[a] Coronal Proton-density fat-suppressed MR image of shoulder showssignal intensity (red arrow) involving supraspinatus tendon; [b] Sagittal Proton-density fat-suppressed MR image of shoulder shows increased signal intensity with soft tissue associated with cystic changes involving acromioclavicular joint (black arrow); [c, d] CT images of shoulder region show bony erosion, subchondral cysts involving acromioclavicular joint (black arrows).

Figure 7

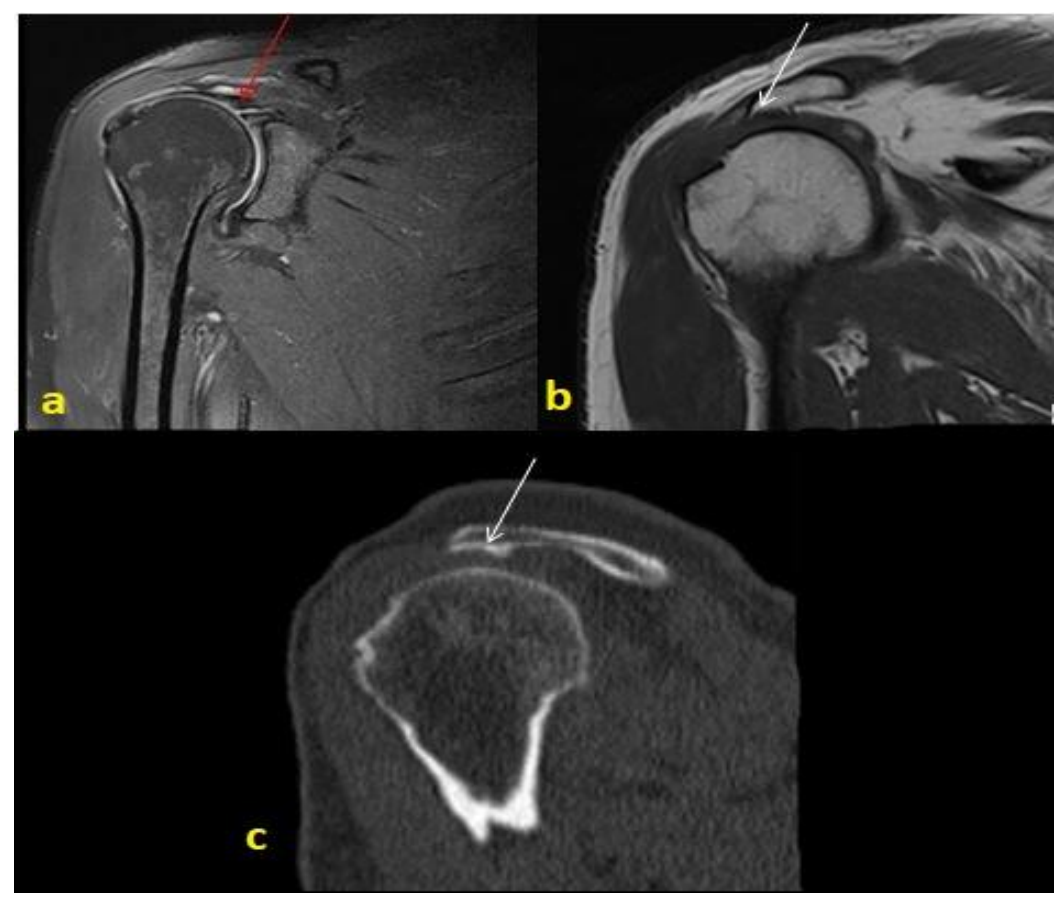

66 year-old male patient with right shoulder pain since 1 year;

[a] Coronal Proton-density fat-suppressed MR image of shoulder shows thinning of supraspinatus tendon with increased signal intensity (red arrow); [b] T1-WEIGHTED MR image of shoulder shows sub-acromial impingement by bony spur (white arrow) over supraspinatus tendon; [c] Sagittal CT image shows a bony spur (white arrow) along the inferior surface of acromion. 


\section{Discussion}

\section{Rotator cuff injuries}

There is an emerging consensus that rotator cuff injury is probably a multifactor process, in which both extrinsic and intrinsic mechanisms have a role. Regardless of pathogenesis, however, it is important to remember that,

(a) The supraspinatus tendon is primarily involved in most instances,

(b) Tears usually begin near the rotator interval,

(c) Partial tears are much more prevalent on the under surface (articular) aspect.

\section{Extrinsic Mechanisms $^{[4]}$}

Acute macrotrauma is an infrequent, yet accepted, mechanism for rotator cuff tear. Repetitive microtrauma, however, is considered by many investigators to be a more relevant factor than acute trauma. According to this theory, stresses on the tendon are manifested by focal -microtears. Codman originally described the -rim rent, which refers to retraction of under surface rotator cuff fibres from the fibro-cartilaginous insertion.

\section{Intrinsic Mechanisms $^{[4]}$}

The role of rotator cuff vascularity in the pathogenesis of tears remains a matter of debate. Supporters of the mechanism of cuff degeneration (tendinopathy) cite supportive histologic findings that are commonly observed with cuff pathologic conditions. Degenerative holes are seen in $50 \%$ of cadavers in post-mortem studies. Although frequently asymptomatic, these foci may predispose the rotator cuff to tear by means of low energy mechanics.

\section{Rotator cuff degeneration and tear}

Rotator cuff degeneration can also be a factor in the rotator cuff tears. Supraspinatus contains a critical zone approximately $1 \mathrm{~cm}$ medial to its insertion which is relatively less perfused area. This area on the articular surface is more prone to the tear ${ }^{[5,6]}$. Generally the rotator cuff tears occur in combination of pathology where the traumatic, impingement and degenerative factors work together. Traumatic tears are more commonly larger in size and involve the multiple tendons ${ }^{[7]}$.
Rotator cuff injuries are well depicted using MRI. Conventional CT lacks the tissue contrast for visualisation of the rotator cuff.

MRI changes depend upon the extent of tears and associated injuries.

Rotator cuff tears can be classified into partial and complete tears.

Partial tears do not involve the entire thickness of the cuff. These tears are common and can be classified into different ways. Partial thickness tears can be classified according to the depth of the tendon thickness affected ${ }^{[8,9]}$. Partial thickness can be Grade I i.e. less than $3 \mathrm{~mm}$ in depth, Grade II i.e. 3 to $6 \mathrm{~mm}$ in depth or Grade III i.e. greater than $6 \mathrm{~mm}$ in depth. Partial thickness tears can be classified according to the location. Partial tears can be on the bursal surface of the tendon, on the articular surface of the tendon or can be intrasubstance ${ }^{[10]}$.

MRI signs of the partial tears are seen as altered zones of sagittal intensity. Partial tears are seen as partial thickness areas of increased signal intensity on proton density fat saturated sequence with corresponding areas showing relatively further increased signal intensity on T2-weighted fat saturated images. Fat suppressed -T2 weighted sequences or the STIR images make the lesions more conspicuous. MR arthrography can improve the diagnosis of the partial articular surface tears [11]. Partial rotator cuff tears can occur as isolated pathology or can be a part of the spectrum of the instability.

A full thickness tear is defined as tear which extends from the articular surface to the bursal surface. Codman defined a complete tear as the one which involves entire thickness of the tendon and the surrounding capsule resulting in communication between subdeltoid bursa and joint cavity. Most of the full thickness tears involve the supraspinatus with subscapularis and infraspinatus involvement if the tears become large and massive.

Conventional MRI can accurately diagnose the complete tears. Complete tear signs can be defined into the direct and indirect signs. Tendon 
discontinuity and fluid signal intensity extending through the entire thickness of the tendon is the most commonly seen MRI feature ${ }^{[12]}$.Tendon retraction can also be present in the larger tears. Secondary signs that can be seen in the complete tears include loss of bursal fat plane, fluid in the sub acromial bursae, muscle atrophy and cysts in the muscle or acromio-clavicular joint, superior migration of the humeral head. MRI shows high level of sensitivity in the diagnosis of the complete tears.

Conventional CT imaging is somewhat limited in evaluation of the rotator cuff. It can be pathognomonic for a chronic tear if there is elevation of the humeral head with either acromiohumeral articulation or an acromio-humeral distance of $7 \mathrm{~mm}$ or less, but this is not commonly seen in patients with acute rotator cuff tears.

Tendinosis is used to define the tendon injury without any tendon defect MRI is a sensitive modality for the diagnosis of the rotator cuff tendinosis. MRI signal intensity changes without surface involvement are typical for tendinosis. Tendons often appear thickened with increase signal intensity on $\mathrm{T} 1$ weighted and proton density sequences with no or little signal intensity increased T2 weighted sequence ${ }^{[13,14]}$. Signal intensity in the tendinosis is very commonly seen in the critical zone which lies $1 \mathrm{~cm}$ proximal to the tendon insertion. Tendinosis needs to be differentiated from the magic angle artefact and the partial volume effects.

\section{Rotator cuff injuries and associations}

Neer et $\mathrm{al}^{[4]}$ proposed the extrinsic theory of rotator cuff injury. This theory proposed that hypertrophic changes in the acromion causes impingement of the sub-acromial subdeltoid bursa and rotator cuff muscle primarily supraspinatus. Intrinsic theory on the other hand explains tendon tears as a result of the tendon degeneration. Degenerative partial thickness tears allow superior migration of the humeral head and leads to abrasion of the supraspinatus tendon. Other possible causes of the rotator cuff disease include the overuse and fatigue of the scapular stabilizers, glenohumeral instability which may lead to the impingement.

Rotator cuff injuries can also occur secondary to the external factors like trauma. Rotator cuff tears are commonly associated with anterior instability. Trauma can be acute or chronic. However most of the tears are chronic with lesser cases occurring in the acute settings.

\section{Rotator cuff impingement}

Impingement can be divided into primary extrinsic impingement, secondary extrinsic impingement (secondary to the instability), internal impingement and subcoracoid impingement.

Primary impingement is due to decreased space for the supraspinatus muscle beneath the coracoacromial arch. Neer et al ${ }^{[4]}$ stated that about 95\% of the rotator cuff tears occur as a result of the chronic impingement beneath the arch. The space below the arch is defined by three things: acromion superiorly, the coraco-acromial ligament supero-medially and coracoid process anteriorly. Variation in any of the three can cause impingement ${ }^{[12]}$.

The acromion morphology plays an important role in supraspinatus injury. Bigliani and colleagues ${ }^{[15]}$ described three types of the acromion based on the shape.

Secondary impingement is seen in relation to the glenohumeral instability. It is seen in people requiring overhead motion. The tears in the anterior labrum and abnormalities in the inferior glenohumeral ligament are most commonly related to the rotator cuff injuries. The anterior labral injury leads to the anterior and superior subluxation of the humeral head which leads to the impingement of the rotator cuff against the acromion.

\section{Acromial morphology and impingement}

The shape of the acromion process may be the single most important anatomic factor in cases of impingement. Acromial features associated with impingement include anterior and lateral spurs, a hooked anterior under surface of the acromion process and lateral down sloping of the acromion. 
Clinical significance of acromion morphology was first described by Bigliani and Morrison in 1987 [16].

Acromial morphology has been classified into three different types by Bigliani ${ }^{[15]}$.

Type I - flat/straight undersurface.

Type II - smooth curved inferior surface.

Type III - Anterior hook/beak.

The slope of the acromion is another factor which can be related to the impingement. Sloping of the acromion either in the upward or downward direction can decrease the space for supraspinatus tendon can cause damage to the tendon.

The hooked acromion can be congenital or acquired. Some researchers believe that type III anteriorly hooked configuration increases in frequency with increasing age ${ }^{[17]}$. The study by Getz et al ${ }^{[18]}$ showed that type III acromion was more common in males and that acromial morphology was symmetric in $70.7 \%$ of 191 pairs of cadaveric acromion without an increased incidence of subacromial enthesophytes in patients with type II and type III acromion. In one study that looked at acromial morphology on oblique sagittal MR images, patients with rotator cuff tears had a significantly increased prevalence of hooked acromion compared with control patients $(62 \%$ versus $13 \%, \mathrm{p}<0.001)$ and there was a greater prevalence of hooked acromion in the group with impingement $(30 \%, \mathrm{p}=0.17)^{[5]}$.

\section{Acromio-humeral distance}

Saupe et al. ${ }^{[2]}$ showed that more than $90 \%$ of patients with an acromiohumeral distance of $\leq 7$ $\mathrm{mm}$ had a full-thickness tear of the supraspinatus tendon and $67 \%$ had a full-thickness tear of the infraspinatus tendon. However, these authors caution "that reduced acromiohumeral distance on conventional radiographs gives information about the integrity of the rotator cuff but cannot be used as a single criterion for surgical decision making in rotator cuff repair."

\section{Acromio-clavicular joint arthritis and impingement}

Primary osteoarthritis more commonly affects the AC joint than glenohumeral joint. Computed tomography is preferred when evaluating arthritic osseous changes of the AC joint such as joint narrowing, erosions, and subchondral cysts. Magnetic resonance imaging has the ability to detect capsular hypertrophy, effusions, and subchondral edema ${ }^{[19]}$.

Seven different single parameters associated with osteoarthritis of the acromio-clavicular joint: cranial and caudal osteophytes, subchondral cysts, subchondral irregularities, bone marrow edema in the distal acromion or clavicle, joint effusion, and capsular hypertrophy ${ }^{[4]}$. Degeneration of acromioclavicular (AC) joints, with associated inferiorly oriented osteophytes, callus, or capsule hypertrophy, can markedly narrow the subacromial space and causes impingement over supraspinatus tendon. Our study proved significance of acromioclavicular arthritis as a significant factor for supraspinatus tears and tendinosis.

\section{Conclusion}

The purpose of our study was to evaluate supraspinatus tears in association with acromion morphology and acromio-clavicular joint changes using CT and MRI.

Acromio-clavicular joint arthritis/ degeneration showed strong association with supraspinatus tears and tendinosis.

Decreased acromio-humeral distance has been associated with rotator cuff injuries especially supraspinatus tendon injuries. As the distance reduces, chances of rotator cuff injury increase. Type I and type II acromion morphology was most commonly observed. Supraspinatus tears were more commonly seen in type II and type III acromion with type III acromion showing strong association with it.

\section{References}

1. Mohamed RE, Abo-sheisha DM. Assessment of acromial morphology in association with rotator cuff tear using magnetic resonance imaging. The Egyptian Journal of Radiology 
and Nuclear Medicine 2014 Mar;45(1).169180.

2. Saupe N, Pfirrmann CWA, Schmid MR, Jost B, Werner CML, Zanetti M. Association Between Rotator Cuff Abnormalities and Reduced Acromiohumeral Distance. AJR AJR Am J Roentgenol.2006 Aug;187(2):376-382.

3. Needell SD, Zlatkin MB, Sher JS, Murphy BJ, Uribe JW. MR imaging of the rotator cuff: peritendinous and bone abnormalities in an asymptomatic population. AJR Am J Roentgenol. 1996 Apr;166(4):863-7.

4. Neer CS 2nd. Anterior acromioplasty for the chronic impingement syndrome in the shoulder: a preliminary report. J Bone Joint Surg Am 1972;54(1):41-50.

5. Jobe CM. Posterior superior glenoid impingement: expanded spectrum. Arthroscopy 1995 Oct;1 1(5):530-6.

6. Ribbans WJ, Mitchell R, Taylor GJ Computerized arthrotomography of primary anterior dislocation of the shoulder. J Bone Joint Surg Br.1990 Mar;72(2): 181-5.

7. Ellman H. Diagnosis and treatment of incomplete rotator cuff tears. Clin OrthopRelat Res. 1990 May; (254):64-74.

8. Snyder SJ, Pachelli AF, Del Pizzo W, Friedman MJ, Ferkel RD, Pattee G. Partial thickness rotator cuff tears: results of arthroscopic treatment. Arthroscopy 1991;7 (1):1-7.

9. Morag Y, Jacobson JA, Miller B, De Maeseneer M, Girish G, Jamadar D. MR imaging of rotator cuff injury: what the clinician needs to know. Radiographics. 2006 Jul-Aug;26(4): 1045-65.

10. Meister K, Thesing J, Montgomery WJ, Indelicato PA, Walczak S, Fontenot W. MR arthrography of partial thickness tears of the undersurface of the rotator cuff: an arthroscopic correlation. Skeletal Radiology. 2004 Mar; 33(3): 136-41.
11. Zlatkin MB, Cary JH, Steven N. Shoulder anatomy. In: Zlatkin MB, editor. MRI of the shoulder, $2^{\text {nd }}$ ed. Philadelphia, PA: Lippincott Williams \& Wilkins; 2003.p.89 -91.

12. Kassarjian A, Bencardino JT, Palmer WE. Radiol Clin North Am. 2006 Jul; 44(4):50323, vii-viii.

13. Kjellin I, Ho CP, Cervilla V, Haghighi P, Kerr $\mathrm{R}$, Vangness $\mathrm{CT}$ et al. Alterations in supraspinatus tendon at MR imaging : correlation with histopathologic findings in cadavers. Radiology. 1991 Dec;181(3):837-41.

14. Sein ML, Walton J, Linklater J, et al. Reliability of MRI assessment of supraspinatus tendinopathy. Br J Sports Med. 2007; 41(8):e9.

15. Bigliani LU, Morrison DS, April EW. The morphology of the acromion and rotator cuff impingement (abstr). Orthop Trans 1986; 10:228.

16. Morrison DS, Biglani LU. The clinical significance of variations in acromial morphology (abstr). Orthop Trans. 1987; 11:234.

17. Ogata S, Uhthoff HK. Acromial enthesopathy and rotator cuff tear: a radiologic and histologic postmortem investigation of the coracoacromial arch. Clin OrthopRelat Res. 1990 May;(254):39-48.

18. J D Getz, M P Recht, D W Piraino, J P Schils, B M Latimer, L M Jellema, N AObuchowski. Acromial morphology: relation to sex, age, symmetry, and subacromial enthesophytes. Radiology 1996 199:3, 737-742.

19. Ernberg, L. A., \& Potter, H. G. (2003). Radiographic evaluation of the acromioclavicular and sternoclavicular joints. Clinics in Sports Medicine, 22(2), 269-273. 